\title{
Surgery of Posterior Communicating Artery Aneurysm
}

\author{
Julius July
}

\subsection{Sign and Symptoms}

Posterior communicating artery (PCoA) aneurysm is the aneurysm that arises at junction of internal carotid (IC) and PCoA. This aneurysm accounts for $15-25 \%$ of all intracranial aneurysm $[1,2]$. If the aneurysm neck arises along the PCoA itself, it's called "true" PCoA aneurysm, and this accounts more or less $1.3 \%$ of all intracranial aneurysm and $6.8 \%$ of all PCoA aneurysm [3].

This aneurysm usually presents with subarachnoid hemorrhage that commonly distributed at the Sylvian fissure and basal cistern but also can be higher up at the Sylvian fissure mimicking the middle cerebral artery rupture. They also can present with oculomotor nerve palsy (34\%) [4], spontaneous subdural hemorrhage (1.72\%) [5], intracerebral hemorrhage at the temporal lobe, and even intraventricular hemorrhage [6].

Although this aneurysm is located at the anterior end of the PCoA, they probably behave more like the posterior circulation aneurysm. ISUIA study finds the rupture risk from PCoA aneurysm is following the posterior circulation $(<7 \mathrm{~mm}$ (2.5\%), 7-12 mm (14.5\%), 13-24 mm (18.4\%), $>25 \mathrm{~mm}(50 \%)$ ), which is higher compared to

\footnotetext{
J. July

Department of Neurosurgery, Faculty of Medicine Universitas Pelita Harapan (UPH), Neuroscience Centre Siloam Hospital Lippo Village,

Tangerang, Indonesia
}

anterior circulation [7]. From the ISUIA study, it seems that the smaller the aneurysm the less likely to rupture. But some study also shows that $87.5 \%$ of ruptured PCoA aneurysm have the size less than $10 \mathrm{~mm}$ and even $40 \%$ of them have the size less than $5 \mathrm{~mm}$ [8].

\subsection{Investigation and Imaging}

Since the era of CT angiography (CTA), it is unnecessary to routinely use the intervention angiography for diagnostic purpose of intracranial aneurysm. Current CTA could provide a three-dimension reconstruction of the vessel, and the imaging is sufficient and very useful for surgical planning.

Several things that the surgeon needs to consider as part of the surgical planning for IC-PCoA aneurysm are the following.

\subsubsection{The Size of the PCoA Itself}

The size of the PCoA needs to be evaluated preoperatively and also its relationship to the aneurysm neck. Sometimes during the clipping, surgeon has to incorporate the PCoA into the clip, although it could be done without the risk of morbidity, but this have to be anticipated prior to surgery. Preoperative evaluation becomes very essential to have a safe surgery. The size of the 
PCoA can range from very small (not visualized on the CTA) to a large artery as big as P2 segment of posterior cerebellar artery (PCA), and usually the P1 segment of the same side is hypoplastic. Such condition is known as fetal PCoA (Fig. 11.1). The surgeon should not compromise such PCoA during the clipping because the major supply to the P2 is mainly coming from the IC. The surgeon has to take this information for

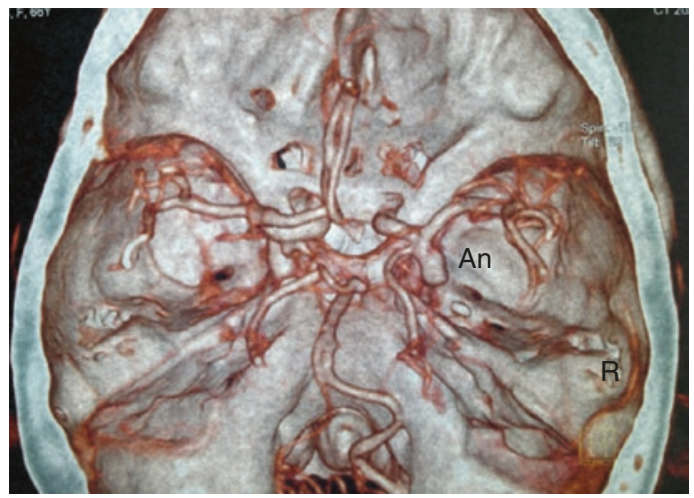

Fig. 11.1 3D reconstruction CTA female 66 years old shows right (R) IC-PCoA aneurysm (An) with ipsilateral fetal PCoA variant. Notice that the P1 segment ipsilateral is hypoplastic; PCoA diameter is as big as P2 segment. The main feeder of right $\mathrm{P} 2$ segment is coming from right IC the surgical planning, including the decision to choose open surgery over the endovascular technique.

\subsubsection{The Direction of Aneurysm Dome}

Simplified classification of the PCoA aneurysm based on dome direction, modified from previous published study $[9,10]$, is divided into two groups, lateral and medial. The classification is based on the line that is drawn parallel with the sagittal plane and the other perpendicular line horizontally. The cross of both lines is located at the IC-PCoA junction (Fig. 11.2). It is not an absolute line and only used as an additional consideration while planning for surgery.

Lateral direction of the dome has higher rupture rate, and try to avoid temporal lobe retraction in the beginning of dissection. The surgeon may apply the temporal retraction gently, after he/she exposes the IC. Anterolateral direction of the dome is often blocking our view to the PCoA origin. Special precaution is needed for such

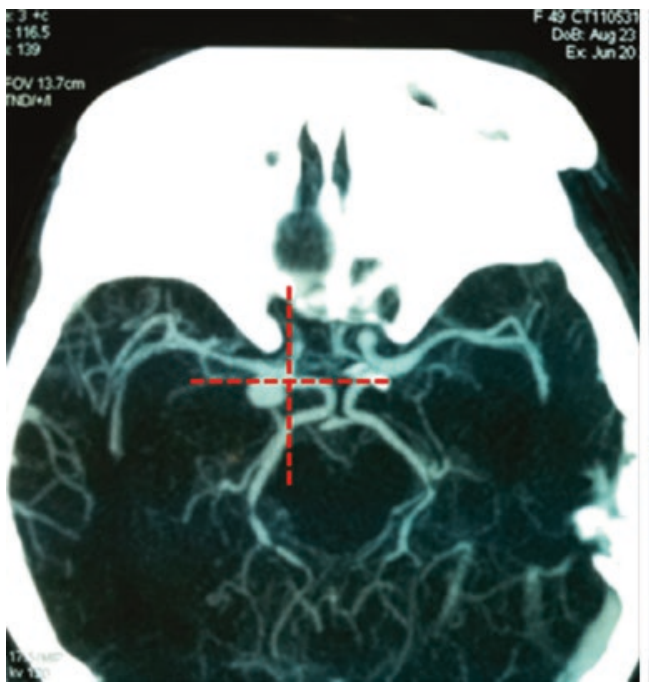

Fig. 11.2 CT angiography of two patients. Left: female 49 years old, with right IC-PCoA aneurysm, dome projecting to lateral and have fetal PCoA bilaterally, and presented with subarachnoid hemorrhage. Right: Female

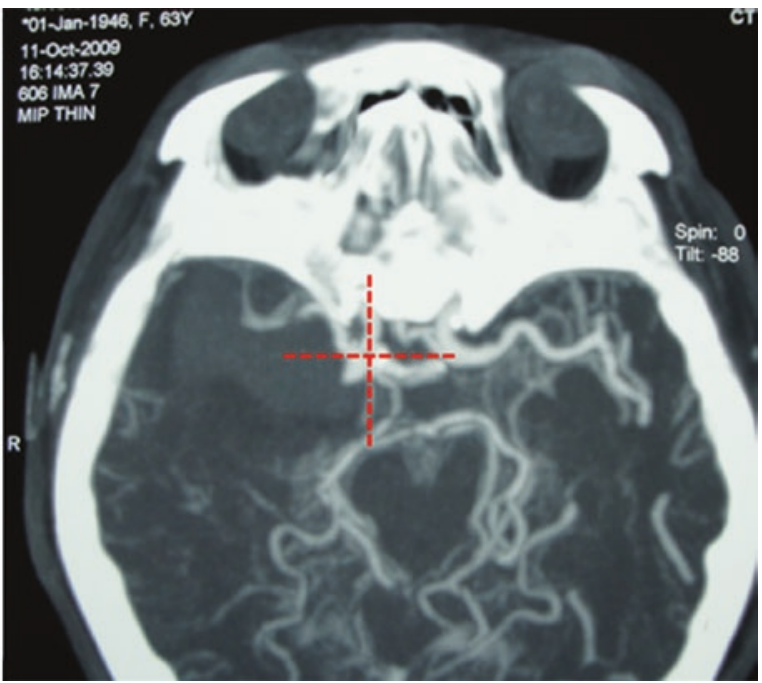

49 years old, with right IC-PCoA aneurysm, dome projecting to lateral, and presented with temporal ICH (intracerebral hemorrhage) and required urgent surgery 
case because some variation of branches from PCoA are supplying important structure such as optic chiasm, oculomotor nerve, mammillary body, tuber cinereum, cerebral crura, ventral thalamus, and rostral portion of the caudate nucleus $[3,10]$.

\subsubsection{Anterior Clinoidectomy}

Anterior clinoidectomy is not necessarily done routinely. A study by a Korean group, looking at the anterior clinoidectomy for IC-PCoA aneurysm, found that only 6 of 94 cases require anterior clinoidectomy [11]. They found three factors based on imaging study (CTA and four vessels angiography) that may help surgeon to estimate whether anterior clinoidectomy is necessary. The distance between aneurysm neck and anterior clinoid process may serve as a simple measure for clinical setting. In the study, the group that requires anterior clinoidectomy (6 patients) was shorter (mean $4.4 \pm 0.7 \mathrm{~mm}$, median $4.7 \mathrm{~mm}$ ), and the group without anterior cinoidectomy (88 patients) was longer (mean 7.2 \pm 1.4 ). Statistically, surgeon should consider anterior clinoidectomy if the distance between the aneurysm neck and anterior clinoid process is less than $4.7 \mathrm{~mm}$. It is rarely required if the distance is more than $5.8 \mathrm{~mm}$ (Fig. 11.3). If the distance is less than $4.7 \mathrm{~mm}$, often the aneurysm neck is covered by the anterior clinoid process, or it blocks the clip while the clipping attempt.

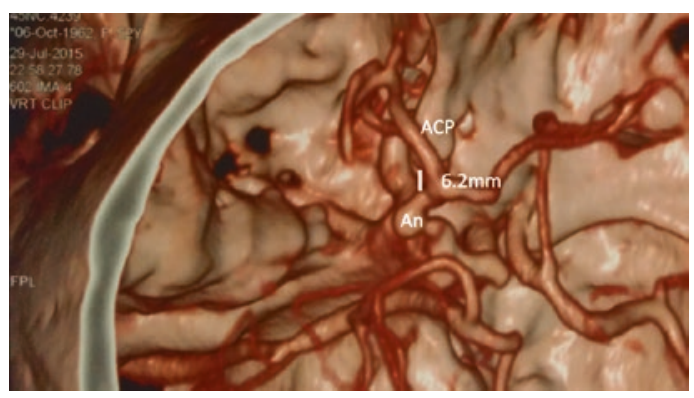

Fig. 11.3 CTA of 52 years old female with left PCoA, the distance of anterior clinoid process (ACP), and the proximal neck of the IC-PCoA aneurysm (An) is $6.2 \mathrm{~mm}$ (white line). In this case, we don't need the anterior clinoidectomy

\subsection{Steps of the Surgery}

\subsubsection{Position, Skin Incision, and Craniotomy}

Patient head should be positioned in order the surgical view and trajectory of the dissection are almost perpendicular to the direction of the dome. This position should allow the surgeon to approach the neck of the aneurysm from the opposite direction of the aneurysm dome/fundus (Fig. 11.4). The head should be elevated $30-45^{\circ}$ above the heart to enhance the brain relaxation because of optimal venous return from intracranial.

The head is fixed with either Sugita or Mayfield or other system of head holder. The skin incision and the craniotomy are planned according to surgeon preference, either pterional approach, lateral supraorbital approach (LSO), or keyhole supraorbital approach.

\subsubsection{Dural Opening and Dissection}

The dura is opened with curve incision with the base toward the skull base and hold with tenting suture silk 3/0 round needle (Fig. 11.5a). The brain is covered by a layer of wet surgicel or cottonoid. Author prefers to use the surgicel for some reasons; when the surgicel is wet, it provides a smooth layer of protection to the brain, and when we remove it, it does not adhere to the brain (some

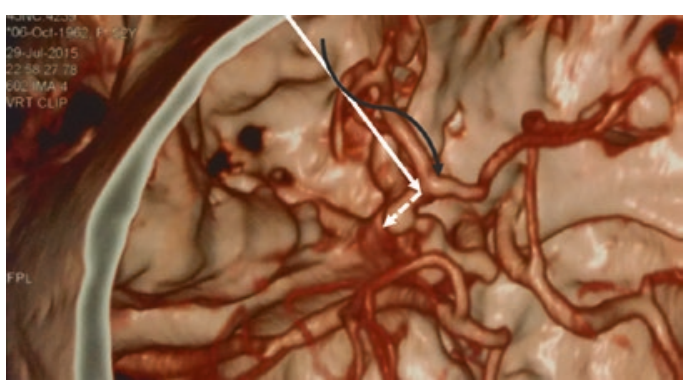

Fig. 11.4 Same patient on Fig. 11.3; the white arrow shows the surgical trajectory that is perpendicular to the direction of the dome (dash arrow). The dissection should avoid the dome of aneurysm and approaching the neck from the opposite direction of the dome 

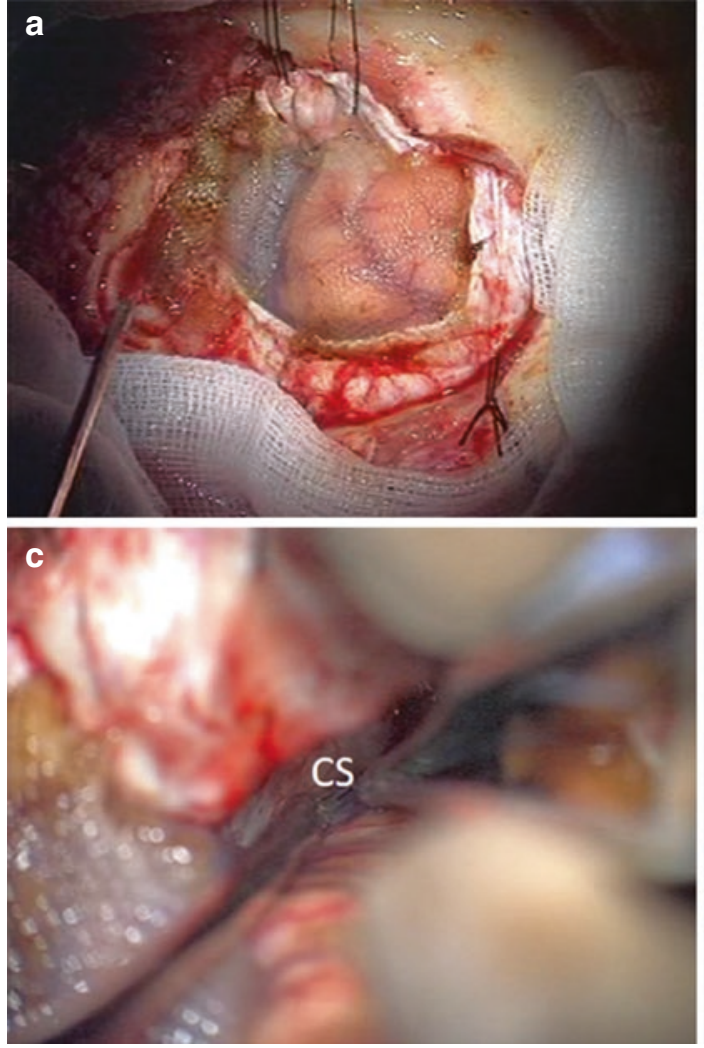

Fig. 11.5 (a) After the craniotomy, the dura is opened with curve shape toward the skull base, and it is tented with silk suture to the muscle. The brain is covered with a wet surgicel. (b) Gently retract the brain and try to reach the chiasmatic cistern, open the arachnoid with micro-

cottonoid may adhere to the brain tissue and may cause bleed when we try to remove it). Firstly, try to reach the chiasmatic cistern and open the arachnoid with micro-scissor (Fig. 11.5b). Once it opened, release the CSF, and the brain will slowly relax, and surgeon will get more space for more dissection (Fig. 11.5c). Always try not to pull anything, and it is better to do sharp dissection all the time. Pulling will increase the chance of premature rupture of the aneurysm.

It is a basic principle to avoid the dome until the surgeon is able to secure the parent artery and the neck of the aneurysm (Fig. 11.5d). A study looking at the risk of intraoperative rupture found that IC-PCoA aneurysm had the second highest rate of rupture $(9.3 \%)$ after the anterior communicating artery (AComA) aneurysm, among the anterior circulation aneurysms [12].
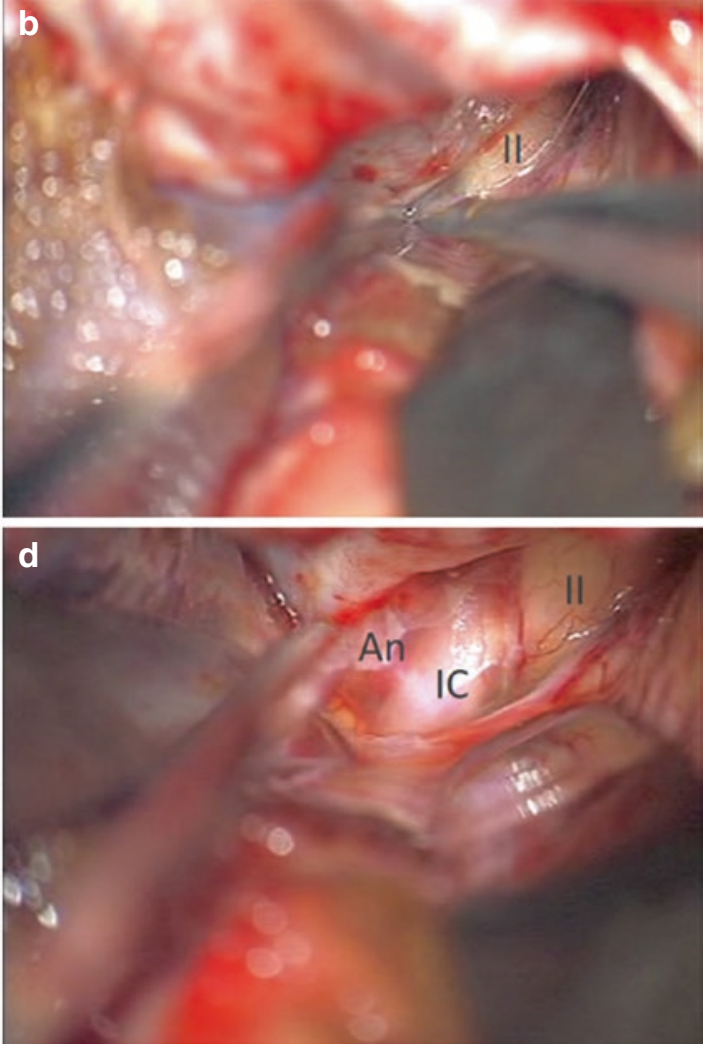

scissor, and release the CSF. (c) The CSF is released and the surgeon will get more working space. (d) Sharply dissect the arachnoid, and identify the optic nerve (chiasm) and internal carotid artery (IC), and we usually will see part of the aneurysm (An)

After the surgeon exposes the parent artery and the neck, he/she may continue dissection to identify the PCoA itself, release the neck from the surrounding arachnoid, and identify the anterior choroidal artery. The surgeon has to minimize the manipulation to the dome of the aneurysm while doing the dissection (Fig. 11.6). Sometimes we could find more than one anterior choroidal artery. After we identify all nearby structure, then the clip may be applied with both legs of the clip adequately squeezing the aneurysm neck. It is important to check all nearby branches are patent. Sometimes, the surgeon may open the lamina terminalis if there is a hydrocephalus on the CT scan.

Nowadays we could check the patency of the branches, the complete clipped of aneurysm, by using the indocyanine green (ICG), but we need to have a special microscope. 


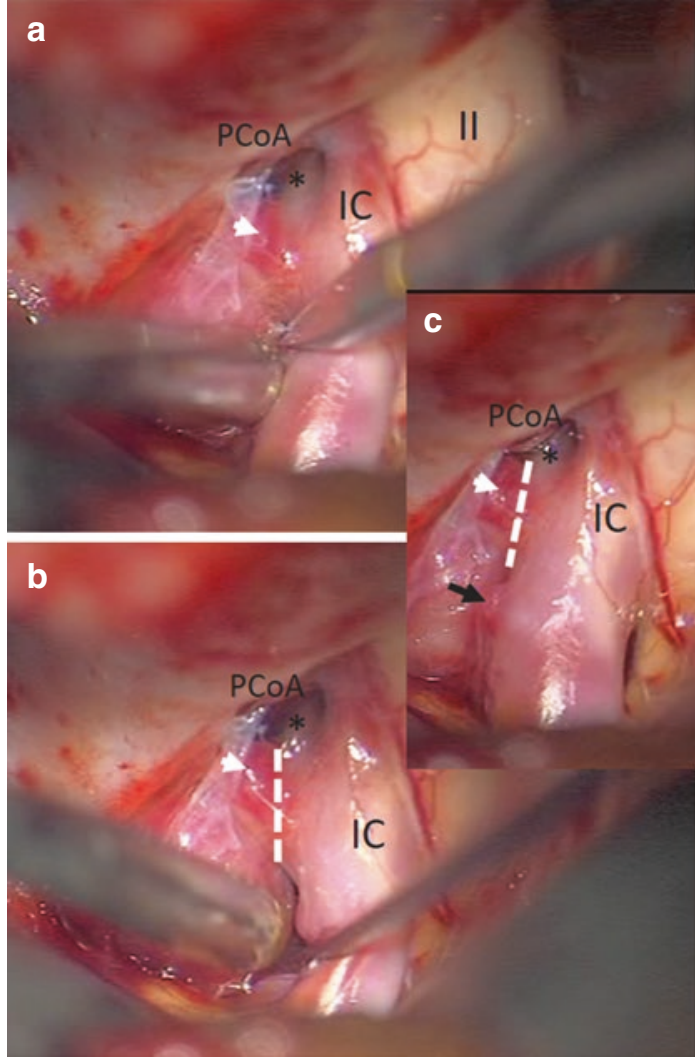

Fig. 11.6 (a-c) The sharp dissection exposes the internal carotid artery (IC), optic nerve (II), PCoA (asterisk), and the neck of the aneurysm (dash line); sometimes we found the bleb on the aneurysm (white arrow) and the anterior

\subsubsection{Closing}

It is better to close the dura mater with watertight closure, tenting the dura mater against the craniotomy edge and using subcutaneous vacuum drain for $24 \mathrm{~h}$. All this effort will minimize the complication and enhance the wound healing.

\subsection{Expert Opinion/Suggestion to Avoid Complication}

All aspect of surgery is important, starting from the planning based on the investigation and imaging study until the postoperative care. During the preparation of surgery, we need a good anesthetic team that will anesthetize the patient without blood pressure (BP) fluctuation. Injection of local d

II

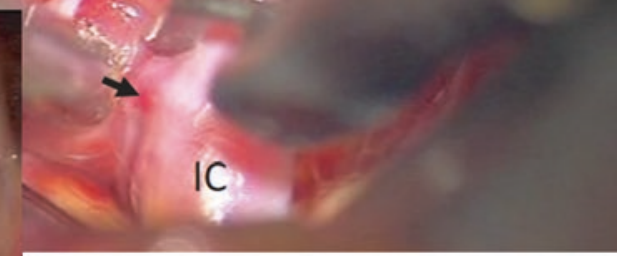

e

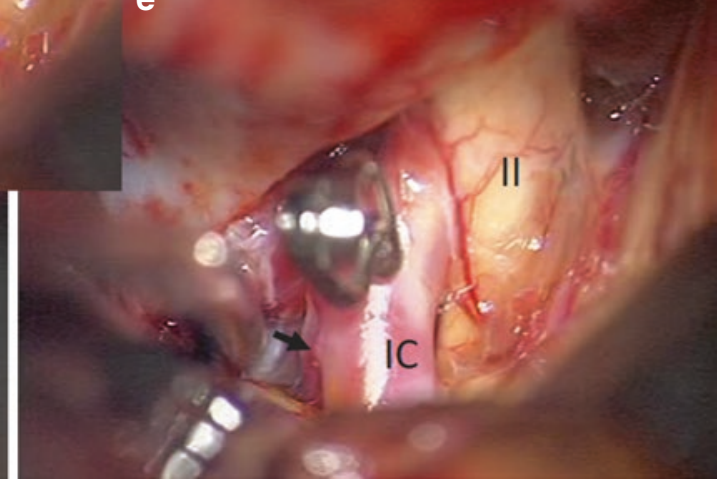

choroidal artery (black arrow). (d) The clip is slowly applied to the aneurysm; in such case we choose bayonet clip. (e) After the clip application, we should make sure the anterior choroidal artery and $\mathrm{PCOA}$ are patent

anesthetic at the head holder pin site will avoid pain, thus no BP changes.

Head positioning is very important, not only for surgical trajectory and view, but it is also contributing to the brain relaxation. The use of mannitol, CSF drainage either at lumbal or ventricular, and $\mathrm{PCO}_{2}$ monitoring are tools that surgeon may use to enhance the brain relaxation. Opening the bone, the duramater and applying the retractor have to be done gently. If we need to remove the anterior clinoid process, try to avoid manipulation of the nearby dura mater, because the dome of the aneurysm might be adhered to the dura mater and manipulation of the dura may cause premature rupture of the aneurysm.

Sharp dissection is a must, and never pull anything during dissection because it may cause remote bleeding that obscures the view and 
causes brain swelling or it may trigger premature rupture of the aneurysm. All the efforts have to be done to prevent premature rupture.

During clip application, simulation is an important part of successful clipping. The surgeon have to always remember that clip revision is very dangerous and often causes catastrophic bleeding and the surgery ends up with higher morbidity and mortality. Always try to avoid clip revision.

Closing is as important as opening. Meticulous closing may reduce the postoperative complication such as epidural hematoma and subdural hematoma.

\subsection{Things to Be Observed and Postoperative Care/ Follow-Up}

After the surgery, hydration is important and also the electrolyte level should be checked. Subarachnoid hemorrhage (SAH) is often followed by cerebral salt wasting syndrome (CSWS) due to the brain natriuretic peptide (BNP) that is released during the SAH. Sodium loss will be followed by water depletion, and the sodium level in the blood will be decreasing. The loss should be replaced and maintained, and sometimes it requires weeks to get it over.

\section{References}

1. Morita A, Kirino T, Hashi K, Aoki N, Fukuhara S, Hashimoto N, et al. The natural course of unruptured cerebral aneurysms in a Japanese cohort. N Engl J Med. 2012;366:2474-82.
2. Ojemann RG, Crowell RM. Surgical management of cerebrovascular disease. 2nd ed. Baltimore: Williams and Wilkins; 1988. Internal carotid artery aneurysms; p. 179-98.

3. He W, Gandhi CD, Quinn J, Karimi R, Prestigiacomo CJ. True aneurysms of the posterior communicating artery: a systematic review and meta-analysis of individual patient data. World Neurosurg. 2011;75:64-72.

4. Soni SR. Aneurysms of the posterior communicating artery and oculomotor paresis. J Neurol Neurosurg Psychiatry. 1974;2013:475-84.

5. Gelabert-Gonzalez M, Iglesias-Pais M, FernandezVilla J. Acute subdural haematoma due to ruptured intracranial aneurysms. Neurosurg Rev. 2004;27:259-62.

6. Golshani K, Ferrell A, Zomorodi A, Smith TP, Britz GW. A review of the management of posterior communicating artery aneurysms in the modern era. Surg Neurol Int. 2010;1:88.

7. Wiebers DO, Whisnant JP, Huston J III, Meissner I, Brown RD Jr, Piepgras DG, et al. Unruptured intracranial aneurysms: natural history, clinical outcome, and risks of surgical and endovascular treatment. Lancet. 2003;362:103-10.

8. Forget TR Jr, Benitez R, Veznedaroglu E, Sharan A, Mitchell W, Silva M, et al. A review of size and location of ruptured intracranial aneurysms. Neurosurgery. 2001;49:1322-5.

9. Fukuda H, Hayashi K, Yoshino K, Koyama T, Lo B, Kurosaki Y, Yamagata S. Impact of aneurysm projection on intraoperative complications during surgical clipping of ruptured posterior communicating artery aneurysms. Neurosurgery. 2016;78(3):381-90.

10. Matsukawa H, Fujii M, Akaike G, Uemura A, Takahashi O, Niimi Y, Shinoda M. Morphological and clinical risk factors for posterior communicating artery aneurysm rupture. J Neurosurg. 2014;120(1):104-10.

11. Park SK, Shin YS, Lim YC, Chung J. Preoperative predictive value of the necessity for anterior clinoidectomy in posterior communicating artery aneurysm clipping. Neurosurgery. 2009;65:281-5.

12. Leipzig TJ, Morgan J, Horner TG, Payner T, Redelman $\mathrm{K}$, Johnson CS. Analysis of intraoperative rupture in the surgical treatment of 1694 saccular aneurysms. Neurosurgery. 2005;56:455-68.

Open Access This chapter is licensed under the terms of the Creative Commons Attribution 4.0 International License (http://creativecommons.org/licenses/by/4.0/), which permits use, sharing, adaptation, distribution and reproduction in any medium or format, as long as you give appropriate credit to the original author(s) and the source, provide a link to the Creative Commons license and indicate if changes were made.

The images or other third party material in this chapter are included in the chapter's Creative Commons license, unless indicated otherwise in a credit line to the material. If material is not included in the chapter's Creative Commons license and your intended use is not permitted by statutory regulation or exceeds the permitted use, you will need to obtain permission directly from the copyright holder. 